\title{
Autoavaliação de saúde e as associações com a síndrome da fragilidade, em idosos atendidos em serviço de geriatria
}

\author{
Self-rated health association with frailty syndrome, in older people treated at a \\ geriatrics service
}

\author{
Dayane Capra de Oliveira' ${ }^{\text {(D) Giovana Sposito }}{ }^{2}$, Vanessa Abreu da Silva ${ }^{3}$ (D), Daniel Eduardo da Cunha Leme ${ }^{4}$ (D), \\ María José D'Elboux ${ }^{5}$
}

Citación: Oliveira DC, Sposito G, Silva VA, Leme DEC, D’Elboux MJ. Autoavaliação de saúde e as associações com a síndrome da fragilidade, em idosos atendidos em serviço de geriatria. Ustasalud 2019;18:7-16.

Licencia Creative Commons

\section{(c) $(1)(8) \Theta$}

\section{RESUMO}

Objetivo: Analisar a associação entre a autoavaliação de saúde e a síndrome da fragilidade em idosos atendidos no serviço de geriatria. Método: Trata-se de um estudo longitudinal que teve na linha de base a participação de 150 idosos. Após oito anos de estudo, 54 idosos da amostra inicial receberam uma segunda avaliação. Os participantes foram avaliados e reavaliados quanto a autoavaliação de saúde que foi classificada em ruim, mais ou menos e boa, assim como, pelo fenótipo da fragilidade identificada por cinco componentes: fraqueza, lentidão, baixo nível de atividade física, exaustão e perda de peso não intencional. Aqueles com nenhum componente foram classificados como não-frágil, aqueles com um ou dois componentes foram classificados como pré-frágeis e aqueles com três ou mais componentes foram considerados frágeis. Para comparar as variáveis de autoavaliação de saúde (ruim, mais ou menos ou boa); classificação da fragilidade (não-frágil, pré-frágil e frágil) e componentes da fragilidade (fraqueza, lentidão, baixo nível de atividade física, exaustão e perda de peso não intencional), foram realizados os testes de qui-quadrado e exato de Fisher. Resultados: A autoavaliação de saúde "ruim" associou-se com os componentes "lentidão" e "exaustão" com maior frequência $(87,5 \%)$ e $(100,0 \%)$, respectivamente $(p<0,05)$. Conclusão: A relação entre medidas subjetivas em saúde e a síndrome da fragilidade prevalente nos idosos, avigora a boa concordância da subjetividade em saúde e condições clínicas capaz de mostrar a relevância de instrumentos que consideram a percepção individual do estado de saúde junto ao rastreio de vulnerabilidade nesta população.

Palavras-chave: Saúde do idoso, autoavaliação, idoso fragilizado, assistência ambulatorial.

\begin{abstract}
Objective: To analyze the association between self-rated health and the frailty syndrome in the elderly assisted in a geriatric service. Method: This longitudinal study had the participation of 150 elderly people at the baseline. After eight years of study, 54 elderly people from the initial sample received a second evaluation. Participants were assessed and reassessed for their self-rated health, which was classified as poor, more or less, and good, as well as for the frailty phenotype identified by five components: weakness, slowness, low level of physical activity, exhaustion and non-weight loss intentional. Those with no components were classified as non-frail, those with one or two components were classified as pre-frail and those with three or more components were considered frail. To compare the variables of self-rated health (poor, more or less or good); frailty classification (non-frail, pre-frail and frail) and frailty components (weakness, slowness, low level of physical activity, exhaustion and unintentional weight loss), chi-square and Fisher's exact tests were performed. Results: Self-rated "poor" health was associated with the components "slowness" and "exhaustion" ( $p$ <.05) more frequently $(87.5 \%)$ and $(100.0 \%)$, respectively. Conclusion: The relationship between subjective measures in health and the frailty syndrome prevalent in the elderly strengthens the good agreement of subjectivity in health and clinical conditions capable of showing the relevance of instruments that consider the individual perception of health status along with the vulnerability screening in this population.
\end{abstract}

Keywords: Health of the elderly; self-assessment; frail elderly; ambulatory care
1 Fisioterapeuta, doutoranda do Programa de Pós-Graduação em Fisioterapia da Universidade Federal de São Carlos.

2 Fisioterapeuta, doutora do Programa de Pós-Graduação em Gerontologia da Faculdade de Ciências Médicas da Universidade Estadual de Campinas.

3 Enfermeira, doutora do Programa de Pós-Graduação em Enfermagem da Faculdade de Enfermagem da Universidade Estadual de Campinas.

4 Fisioterapeuta, doutorando do Programa de Pós-Graduação em Gerontologia da Faculdade de Ciências Médicas da Universidade Estadual de Campinas.

5 Livre docente, professora colaboradora na Faculdade de Ciências Medicas da Universidade Estadual de Campinas.

\section{Autor de correspondencia:}

Dayane Capra de Oliveira

Correo electrónico:

dayacapra@gmail.com
Recibido para publicación: 8 de abril de 2019 Aceptado para publicación:

30 de noviembre de 2019 


\section{INTRODUÇÃO}

A autoavaliação de saúde é uma medida subjetiva de fácil aplicação, alta validade e confiabilidade. Refere-se a uma pergunta simples, sobre como o avaliado considera a sua própria saúde, com resposta escalar "excelente", "boa", "regular" ou "ruim". Este indicador é citado em pesquisas epidemiológicas realizadas no âmbito nacional e internacional como preditor de morbidades, inatividade física, perdas funcionais e mortalidade ${ }^{1-3}$.

$\mathrm{Na}$ geriatria e gerontologia, a autoavaliação de saúde tem recebido crescente atenção como um parâmetro de avaliação da saúde do idoso ${ }^{(2-3)}$. Estudos populacionais realizados com pessoas idosas mostraram associação entre percepção de saúde e perdas funcionais ${ }^{4}$, doenças crônicas ${ }^{5}$ e mortalidade ${ }^{6,7}$. Idosos que percebem sua própria saúde como ruim, apresentam riscos para desfechos negativos em saúde ${ }^{8}$.

Ademais, a literatura ressalta a relação entre autoavaliação de saúde e medidas objetivas de desempenho funcional em idosos comunitários. Especificamente, a autoavaliação negativa de saúde esteve associada com fraqueza muscular mensurada pela baixa força de preensão manual. Evidencia-se a importância de medidas subjetivas simples de caráter valorativo, no rastreio de vulnerabilidades?

No contexto das condições clínicas prevalentes na velhice, a fragilidade destaca-se como síndrome geriátrica associada com incapacidades, morbidades e mortalidade ${ }^{10}$. Esta condição clínica pode ser identificada por cinco componentes físicos descritos no estudo original do fenótipo de fragilidade ${ }^{11}$ - perda de peso não intencional, exaustão, fraqueza, lentidão e baixo nível de atividade física.

A relação entre autoavaliação de saúde e componentes da fragilidade pode estar presente na medida em que a síndrome, em sua multidimensionalidade, resulta em alterações físicas e psicológicas, as quais influenciam a redução na participação social e percepção de bem-estar do idoso, mas ainda pouco se sabe a respeito dessa relação, principalmente em grupos específicos de idosos vulneráveis atendidos na alta complexidade ${ }^{9,12}$.
Isto posto, o presente estudo teve como objetivo avaliar a associação entre a autoavaliação de saúde e os componentes do fenótipo de fragilidade, em idosos assistidos em serviço ambulatorial de Geriatria.

\section{MÉTODOS}

Trata-se de um estudo longitudinal realizado em dois momentos com idosos assistidos no Ambulatório de Geriatria do Hospital de Clínicas (HC) da Universidade Estadual de Campinas (Unicamp), Campinas, SP.

O Ambulatório de Geriatria da Unicamp é um serviço público de saúde que atende pessoas idosas com 60 anos ou mais de idade. Estes pacientes são encaminhados de outras especialidades médicas do HC-Unicamp e Unidades Básicas de Saúde do município de Campinas-SP e região.

No primeiro momento foi realizada a avaliação dos idosos, efetuada por alunos do Programa de Pós-Graduação em Gerontologia da Unicamp, entre outubro de 2005 a setembro de 2007, e o segundo momento foi realizado a reavaliação que ocorreu entre janeiro a maio, do ano de 2013, totalizando seis anos de estudo para aqueles que foram avaliados em 2005 e oito anos para aqueles avaliados em 2007.

O processo de amostragem deste estudo se deu por conveniência, ou seja, selecionamos uma amostra da população prontamente disponível em nosso ambulatório. Este tipo de amostragem foi considerado neste estudo dada a sua facilidade operacional e o baixo custo.

Para tanto, foram respeitados os seguintes critérios de inclusão: (1) ser usuários do referido Ambulatório de Geriatria; (2) ausência de déficit cognitivo que dificultasse a comunicação verbal e que apresentassem pontuação no Mini Exame do Estado Mental (MEEM) igual ou superior a 13 (para analfabetos), 18 ( 1 a 7 anos de escolaridade), 26 (escolaridade igual ou superior a oito anos $)^{13}$

Realizou-se a "reavaliação" no período de janeiro a maio de 2013, pelos mesmos pesquisadores da fase inicial do estudo. Para tanto, os idosos participantes 
da avaliação ou responsáveis por eles foram contatados via telefonema, para então serem convidados para esta segunda etapa. Considerou-se o período de seguimento do estudo, o período entre a avaliação e a reavaliação de cada paciente.

Desta forma, na linha de base, foram analisados 150 idosos. Destes, 71 evoluíram para óbito, 5 se recusaram em participar da reavaliação, 4 mudaram para cidades distantes do local da pesquisa e 16 idosos não foram localizados, pois havia desatualização dos endereços e/ou telefones de contato. Sendo assim, 54 pacientes foram reavaliados.

Os indivíduos foram avaliados e reavaliados por meio de informações sociodemográficas (idade e sexo), condições de saúde, relato de hospitalizações e quedas nos últimos 12 meses. As condições de saúde foram coletadas pelo autorrelato indicando a presença ou não das doenças crônicas: hipertensão arterial, diabetes, doenças cardiovasculares, osteoartrite, osteoporose, doença renal crônica, Doença Pulmonar Obstrutiva Crônica e depressão. A presença destas doenças foi utilizada como um somatório onde o mais alto número de respostas afirmativas indica maior o número de doenças prevalentes.

A fragilidade, variável de interesse, foi analisada segundo a definição do modelo do fenótipo de fragilidade descrito por Linda Fried e cols. ${ }^{11}$. Neste modelo, a condição de fragilidade é determinada a partir de cinco componentes físicos:

- Perda de peso não intencional no último ano antecedente à pesquisa. Pontuaram positivamente para este componente, os idosos que relataram perda de peso superior a $4,5 \mathrm{Kg}$ ou mais de $5 \%$ do peso corporal.

- Exaustão: avaliada a partir de duas questões extraídas da escala de rastreamento de depressão "The Center for Epidemiologic Studies - Depression" $(C E S-D)^{14}$, e adaptadas para o contexto de avaliação dos idosos brasileiros ${ }^{15}$, são elas: "sentiu que teve que fazer esforço para dar conta das tarefas habituais" e "não conseguiu levar adiante suas coisas". Os idosos que relataram experimentar qualquer um dos sintomas citados, em uma frequência de três ou mais dias durante a semana prévia à pesquisa, pontuaram positivamente para o referido componente de fragilidade.

- Lentidão: avaliada pelo tempo cronometrado em milésimos de segundo, para percorrer em duas tentativas, uma distância de 4,0 metros em passo usual. Os resultados obtidos foram ajustados por sexo e altura, sendo considerado positivo para este componente o tempo de percurso maior ou igual a sete e seis segundos, tanto para homens quanto para mulheres, segundo as recomendações de Guralnik e cols. ${ }^{16}$ e Nakano ${ }^{17}$.

- Fraqueza: avaliada pela força de preensão palmar, utilizado um aparelho dinamômetro isocinético portátil da marca Crown $n^{\circledast}$ com capacidade de $50 \mathrm{Kg}$. Para tanto, o participante foi orientado a permanecer sentado em uma cadeira, com o cotovelo do membro superior dominante flexionado a $90^{\circ}$, segurando o aparelho supracitado durante o teste. Após o comando do pesquisador, o idoso realizou três tentativas de preensão manual com o máximo de força possível apenas com flexão das articulações interfalangeana e a metacarpo-falangeana. Os resultados foram ajustados por sexo e índice de massa corpórea $(\mathrm{IMC})^{18}$ e atribuiu-se pontuação positiva neste componente, os idosos que apresentaram os valores em quilograma-força (Kgf) descritos para homens e mulheres no (Quadro 1).

Quadro 1. Critérios de pontuação para fragilidade na prova de força de preensão manual, segundo sexo e IMC. Campinas, 2014

\begin{tabular}{ll}
\hline \multicolumn{1}{c}{ IMC em Kg/m } & \multicolumn{1}{c}{$\begin{array}{c}\text { Pontos de corte para } \\
\text { Fragilidade em Kgf }\end{array}$} \\
\hline Homens & $\leq 29$ \\
\hline$\leq 24$ & $\leq 30$ \\
24,1 a 26 & $\leq 30$ \\
26,1 a 28 & $\leq 32$ \\
\hline 28 & \\
\hline Mulheres & $\leq 17$ \\
\hline$\leq 23$ & $\leq 17,3$ \\
23,1 a 26 & $\leq 18$ \\
26,1 a 29 & $\leq 21$ \\
\hline 29 & \\
\hline
\end{tabular}

Fonte: Silva (18) 
- Baixo nível de atividade física: foi mensurado pelo autorrelato das seguintes perguntas: $O$ (a) senhor(a) pratica atividades físicas regularmente? Se a resposta fosse afirmativa, quantas vezes na semana? um ou dois dias; dois ou três dias; três ou quatro dias ou maior que quatro dias. A atividade física foi considerada a prática de esportes ativos ou tarefas domésticas realizadas pelo idoso. Em caso de resposta negativa ou de frequência igual a uma ou duas vezes por semana, foi considerado baixo nível de atividade física.

Com base nesses componentes, os idosos foram classificados como "frágeis" quando apresentaram três ou mais componentes, "pré-frágeis" quando apresentaram um ou dois componentes e "não-frágeis" se não apresentaram nenhum componente.

A autoavaliação de saúde, variável desfecho deste estudo, foi mensurada por meio da seguinte pergunta: Como é a sua saúde de um modo geral? Havia três possibilidades de respostas: "ruim", "mais ou menos" "boa". Esta questão foi elaborada com base em estudos multicêntricos e na literatura pesquisada ${ }^{19,20}$.

Foram realizadas análises descritivas com valores de média e desvio padrão, para variáveis numéricas e valores de proporção para variáveis categóricas. Para comparação das variáveis relacionadas à autoavaliação de saúde (ruim, mais ou menos ou boa); classificação da fragilidade (não-frágil, pré-frágil e frágil) e componentes da fragilidade (perda de peso não intencional; exaustão; lentidão; fraqueza e baixo nível de atividade física), foram realizados os testes de qui-quadrado e exato de Fisher, entre as fases de avaliação e reavaliação. Para todos os testes estatísticos, o nível de significância adotado foi de $5 \%$, ou seja, $p<0,05$. Todos os dados foram analisados por meio do programa Statistical Analysis System for Windows ${ }^{\ominus}$ (SAS System).

Este estudo foi aprovado pelo Comitê de Ética em Pesquisa da Faculdade de Ciências Médicas da Unicamp, como um adendo ao projeto de pesquisa principal denominado "Qualidade de vida em idosos: indicadores de fragilidade e de bem-estar subjetivo", no qual tem o parecer $\mathrm{n}^{\circ} 240 / 2003$. Ademais foram cumpridos os termos da Resolução no 466/12 de 12 de dezembro de 2012 do Conselho Nacional de Saúde, do Ministério da Saúde. Todos os participantes assinaram o Termo de Consentimento Livre e Esclarecido.

\section{RESULTADOS}

Do total de idosos que participaram da avaliação, $64 \%$ eram mulheres, tinham vida conjugal $(46,7 \%)$, além de predomínio de idosas $(43,4 \%)$ na faixa etária entre 70 e 79 anos de idade. Ainda nesta fase, 55,3\% dos participantes foram classificados como frágeis; a média de doenças crônicas foi de 5,43 $\pm 2,5$ e mais da metade $(51,3 \%)$ referiu pelo menos uma queda nos 12 meses prévios ao estudo.

Dos 54 reavaliados, a maioria era mulheres $(66,7 \%)$ e o grupo de octogenárias foi prevalente $(64,1 \%)$. Notou-se alta prevalência de fragilidade $(88,9 \%)$ e os idosos tinham em média 7,33 $\pm 2,3$ doenças crônicas e $59,3 \%$ relataram alguma queda nos 12 meses anteriores à pesquisa (Tabela 1).

Na tabela 2, observa-se que não houve associação estatisticamente significativa entre autoavaliação de saúde e classificação de fragilidade nos dois momentos da pesquisa.

A autoavaliação de saúde "ruim" associou-se com os componentes "lentidão" e "exaustão" com maior frequência $(87,5 \%)$ e $(100,0 \%)$ respectivamente $(\mathrm{p}<0,05)$ (Tabela 3).

\section{DISCUSSÃO}

Este estudo avaliou a associação entre autoavaliação de saúde e componentes da fragilidade em idosos ambulatoriais, examinados em dois momentos com intervalo de até oito anos, compreendido o maior período de seguimento completado entre os anos de 2005 e 2013. Os principais resultados mostraram associação significativa entre autoavaliação de saúde e os componentes "lentidão" e "exaustão", na avaliação e reavaliação.

Em ambas as fases deste estudo, houve maior porcentagem de mulheres. É sabido que a busca pelos ser- 
Tabela 1. Características descritivas dos idosos atendidos em um serviço de geriatria na avaliação e reavaliação final do estudo. Campinas 2005/07 - 2013, Brasil

\begin{tabular}{|c|c|c|}
\hline & $\begin{array}{c}\text { Avaliação } \\
(n=150)\end{array}$ & $\begin{array}{c}\text { Reavaliação } \\
(\mathrm{n}=54)\end{array}$ \\
\hline \multicolumn{3}{|l|}{ Sexo } \\
\hline Homem, \% & 36,0 & 33,3 \\
\hline Mulher, \% & 64,0 & 66,7 \\
\hline \multicolumn{3}{|l|}{ Idade } \\
\hline$<70$ anos, $\%$ & 23,3 & 3,8 \\
\hline 70-79 anos, $\%$ & 43,4 & 32,1 \\
\hline$\geq 80$ anos, $\%$ & 33,3 & 64,1 \\
\hline \multicolumn{3}{|l|}{ Estado conjugal } \\
\hline Casado & 46,7 & 32,7 \\
\hline Viúvo & 42,7 & 53,8 \\
\hline Divorciado & 5,3 & 7,7 \\
\hline Solteiro & 5,3 & 5,8 \\
\hline Hospitalização (sim), \% & 40,3 & 44,2 \\
\hline Quedas (sim), \% & 51,3 & 59,3 \\
\hline Número de doenças, (DP) & $5,43(2,5)$ & $7,33(2,3)$ \\
\hline \multicolumn{3}{|l|}{ Fragilidade } \\
\hline Não-frágil, \% & 2,7 & - \\
\hline Pré-frágil, \% & 42,0 & 11,1 \\
\hline Frágeis, \% & 55,3 & 88,9 \\
\hline \multicolumn{3}{|l|}{ Componentes } \\
\hline Fraqueza (sim), \% & 58,4 & 87,0 \\
\hline Lentidão (sim), \% & 54,7 & 77,8 \\
\hline Baixo nível de atividade física (sim), \% & 80,5 & 92,6 \\
\hline Exaustão (sim), \% & 27,0 & 62,9 \\
\hline Perda de peso não intencional ( $\mathrm{sim}), \%$ & 46,5 & 59,3 \\
\hline
\end{tabular}

Dados expressos em média, desvios padrão e proporção. Preenchidos com hífen (-) representam caselas vazias.

Tabela 2. Autoavaliação de saúde e classificação de fragilidade em idosos ambulatoriais. Campinas-SP, 2005/07 - 2013, Brasil

\begin{tabular}{|c|c|c|c|c|c|c|c|c|}
\hline & \multicolumn{3}{|c|}{ Avaliação $(n=150)$} & \multicolumn{5}{|c|}{ Reavaliação $(\mathrm{n}=54)$} \\
\hline & $\begin{array}{l}\text { Ruim } \\
\text { n (\%) }\end{array}$ & $\begin{array}{c}\text { Mais ou } \\
\text { menos } \\
\text { n (\%) }\end{array}$ & $\begin{array}{c}\text { Boa } \\
\text { n (\%) }\end{array}$ & p-valor & $\begin{array}{l}\text { Ruim } \\
\text { n (\%) }\end{array}$ & $\begin{array}{c}\text { Mais ou } \\
\text { menos } \\
\text { n (\%) }\end{array}$ & $\begin{array}{c}\text { Boa } \\
\text { n (\%) }\end{array}$ & p-valor \\
\hline $\begin{array}{l}\text { Classificação da } \\
\text { fragilidade, \% }\end{array}$ & & & & 0,759 & & & & 0,580 \\
\hline Não frágil & - & $2(2,7)$ & $2(4,3)$ & & - & - & - & \\
\hline Pré-frágil & $9(33,3)$ & $32(43,2)$ & $20(42,5)$ & & - & $5(16,1)$ & $1(6,7)$ & \\
\hline Frágil & $18(66,7)$ & $42(54,1)$ & $25(53,2)$ & & $8(100,0)$ & $26(83,9)$ & $14(93,3)$ & \\
\hline TOTAL & $27(100,0)$ & $76(100,0)$ & $47(100,0)$ & & $8(100,0)$ & $31(100,0)$ & $15(100,0)$ & \\
\hline
\end{tabular}

Significância estatística de p <0,05 (Teste Exato de Fisher). Preenchidos com hífen (-) representam caselas vazias. 
Tabela 3. Autoavaliação de saúde e componentes da fragilidade em idosos ambulatoriais. Campinas-SP, 2005/07 - 2013, Brasil

\begin{tabular}{|c|c|c|c|c|c|c|c|c|}
\hline \multirow{3}{*}{$\begin{array}{l}\text { Componentes da } \\
\text { fragilidade }\end{array}$} & \multicolumn{8}{|c|}{ Autoavaliação de saúde } \\
\hline & \multicolumn{3}{|c|}{ Avaliação (n=150) } & \multicolumn{5}{|c|}{ Reavaliação $(\mathrm{n}=54)$} \\
\hline & $\begin{array}{l}\text { Ruim } \\
\text { n (\%) }\end{array}$ & $\begin{array}{c}\text { Mais ou } \\
\text { menos } \\
\text { n }(\%)\end{array}$ & $\begin{array}{c}\text { Boa } \\
\text { n (\%) }\end{array}$ & $\mathrm{p}$ & $\begin{array}{l}\text { Ruim } \\
\text { n (\%) }\end{array}$ & $\begin{array}{c}\text { Mais ou } \\
\text { menos } \\
\text { n }(\%)\end{array}$ & $\begin{array}{c}\text { Boa } \\
\text { n (\%) }\end{array}$ & p \\
\hline Baixo nível de atividade física & & & & 0,448 & & & & 1,000 \\
\hline Não & $5(18,5)$ & $12(16,2)$ & $12(25,5)$ & & - & $3(9,7)$ & $1(7,2)$ & \\
\hline Sim & $22(81,5)$ & $62(83,8)$ & $35(74,5)$ & & $8(100,0)$ & $28(90,3)$ & $13(92,8)$ & \\
\hline Perda de peso & & & & 0,569 & & & & 0,332 \\
\hline Não & $13(48,1)$ & $37(50,7)$ & $25(59,5)$ & & $2(25,0)$ & $12(38,7)$ & $8(57,1)$ & \\
\hline Sim & $14(51,9)$ & $36(49,3)$ & $17(40,5)$ & & $6(75,0)$ & $19(61,3)$ & $6(42,9)$ & \\
\hline Fraqueza muscular & & & & 0,484 & & & & 0,147 \\
\hline Não & $9(33,3)$ & $34(45,9)$ & $18(39,1)$ & & - & $3(9,7)$ & $4(28,6)$ & \\
\hline Sim & $18(66,7)$ & $40(54,1)$ & $28(60,9)$ & & $8(100,0)$ & $28(90,3)$ & $10(71,4)$ & \\
\hline Lentidão & & & & $0,022^{*}$ & & & & $0,026^{\star}$ \\
\hline Não & $6(22,2)$ & $39(52,7)$ & $23(48,9)$ & & $1(12,5)$ & $6(19,4)$ & $8(53,3)$ & \\
\hline Sim & $21(77,8)$ & $35(47,3)$ & $24(51,1)$ & & $7(87,5)$ & $25(80,6)$ & $7(46,7)$ & \\
\hline Exaustão & & & & $0,006^{*}$ & & & & $0,046^{\star}$ \\
\hline Não & $13(48,1)$ & $58(78,4)$ & $37(78,7)$ & & - & $14(45,2)$ & $6(42,9)$ & \\
\hline Sim & $14(51,9)$ & $16(21,6)$ & $10(21,3)$ & & $8(100,0)$ & $17(54,8)$ & $8(57,1)$ & \\
\hline
\end{tabular}

Significância estatística de p<0,05 (Teste Exato de Fisher). Preenchidos com hífen (-) representam caselas vazias.

viços de saúde é habitualmente feminina, por outro lado a não procura pelos atendimentos em saúde por parte dos idosos do sexo masculino, pode estar relacionada com o papel social construído para o homem, que engloba atributos de competitividade e de robuste $\mathrm{z}^{21}$. Outro fator relevante diz respeito à "feminização" da população, sendo um processo comumente observado na população brasileira ${ }^{22}$.

Além disso, nas idades mais avançadas foi observado o predomínio de mulheres, notadamente na fase de reavaliação. Este resultado é correspondente com os dados de estudos previamente publicados ${ }^{23,24}$, os quais ressaltam sobre a representação feminina na faixa etária acima dos oitenta anos de idade, sendo resultado da maior expectativa de vida das mulheres, que vivem em média oito anos a mais em relação aos homens.

A fragilidade tem sido amplamente investigada em diversos desenhos metodológicos. Especialmente se tratando da operacionalização desta síndrome, os modelos de avaliação que abrangem os domínios fí- sico, social, psicológico e cognitivo são estudados nos últimos anos e mostram-se confiáveis e eficazes para o rastreamento de idosos frágeis inseridos nos diversos contextos de assistência em saúde, na baixa e alta complexidade ${ }^{25}$.

Neste estudo foi utilizado o modelo proposto por Linda Fried e cols. ${ }^{11}$, no qual é constituído por componentes prioritariamente direcionados à avaliação da saúde física do idoso, embasados nas alterações fisiológicas e biológicas do envelhecimento. Dessa forma, foi possível rastrear os pacientes pré-frágeis e frágeis, sobretudo, por meio deste instrumento a alta porcentagem de fragilidade foi observada na amostra estudada, para ambos os períodos (avaliação e reavaliação).

Os idosos que participaram deste estudo eram acompanhados em um serviço de alta complexidade em saúde, e compreende-se que neste tipo de assistência é comum os pacientes apresentarem maiores vulnerabilidades, por exemplo, multimorbidade, incapacidade funcional nas atividades básicas de vida diária 
e atividades instrumentais de vida diária e problemas cognitivos. Esse fato pode justificar o elevado número de frágeis, de modo a corroborar a literatura ${ }^{26}$.

Ainda em relação à fragilidade, notou-se que na reavaliação as idosas, em maioria, apresentaram a síndrome. De outro modo, Freitas e cols. ${ }^{27}$ ao avaliarem 103 idosos assistidos em ambulatório de geriatria de um hospital universitário, notaram que apesar da alta porcentagem de mulheres com condições desfavoráveis de saúde (presença de doenças e incapacidades), tal grupo vulnerável não apresentou maiores índices de fragilidade.

Entretanto, a maior prevalência da síndrome de fragilidade em mulheres é observada na literatura, sendo explicada por condições fisiológicas, dentre elas o menor índice de massa muscular em relação aos homens, assim como condições sociais restritas ao sexo feminino (menor escolaridade, pouca independência econômica, baixo nível econômico e vida social restrita); tais circunstâncias socioeconômicas condizem ao perfil da população idosa atual que vivenciou o menor acesso à educação, menores oportunidades no mercado de trabalho, consequentemente menores rendas e qualidade de vida ${ }^{28}$.

Interessantemente, notou-se a tendência dos idosos avaliarem negativamente a própria saúde e também apresentarem sintomas e/ou sinais físicos como exaustão e lentidão, tendo em conta a diferença significativa entre grupos avaliados ao longo do seguimento. Um estudo elaborado por Melo et al. ${ }^{29}$ mostrou que os idosos ambulatoriais que avaliaram sua própria saúde como "ruim" apresentaram os componentes de fragilidade "exaustão" e "lentidão". Os autores ressaltaram sobre a importância da percepção de saúde positiva e a manutenção do envolvimento social, como condições que amenizam as perdas funcionais e inatividade em grupos vulneráveis.

Nas fases de avaliação e reavaliação, também houve frequência expressiva de idosos que avaliaram a própria saúde como "mais ou menos" e que pontuaram positivamente para os componentes de fragilidade "lentidão" e "exaustão". Corroborando este achado, um estudo populacional ${ }^{30}$ envolvendo 1.220 idosos com comorbidades e incapacidade funcional mostrou que $80 \%$ dos participantes avaliaram sua própria saúde como "regular", e com o avançar da idade as percepções de saúde pioravam. Resultados semelhantes também foram observados no Australian Longitudinal Study of Ageing $(A L S A)^{31}$.

De fato, o avançar da idade relaciona-se com o aumento de morbidades crônicas e incapacidades, e estes fatores influenciam negativamente o bem-estar dos idosos, consequentemente subentende-se que nas idades avançadas a piora da autoavaliação de saúde seja comumente observada ${ }^{32,33}$. Siqueira e cols. ${ }^{34}$, ao compararem amostras diferentes compostas de pessoas adultas e de idosos notaram que aproximadamente dois terços dos idosos longevos (80 anos ou mais de idade) consideram sua própria saúde como regular ou ruim, e entre os jovens este índice decresceu menos da metade.

Mesmo que as autocrenças favoreçam a continuidade das estratégias para superar eventos adversos do envelhecimento, elas perdem força quando os idosos se deparam com situações como dor crônica, fraqueza muscular, fadiga e lentidão. É sugestivo que ao se depararem com situações que sobressaem às suas estratégias de enfrentamento, os idosos formarão julgamento negativo a respeito da saúde. Salienta-se que as autoavaliações de saúde são alimentadas pelos indicadores fisiológicos como dor, fadiga e perda da energia 9 .

Sob outra perspectiva, ora relevante e que mostra outro enfoque da subjetividade em saúde, alguns idosos que pontuaram positivamente para lentidão ou exaustão apresentaram a autoavaliação de saúde "boa". A justificativa da avaliação positiva da própria saúde entre pré-frágeis ou frágeis é mencionada por Ebrahimi e cols. ${ }^{35}$, ao explicarem que idosos mais vulneráveis suportam melhor o sofrimento ao longo da vida, fragilidade e adversidades.

Essa hipótese também é sustentada por Eloranta e cols. ${ }^{36}$, quando afirmaram que pessoas idosas apresentam autoavaliação de saúde boa, mesmo quando são portadores de doenças e incapacidades físicas. Borim e cols. ${ }^{12}$ acrescentaram que avaliações otimistas entre os idosos estão presentes sempre quando eles são capazes de ajustar suas expectativas ou enquanto não fo- 
ram acometidos por problemas de saúde mais graves. Assim, eles conseguem lidar melhor com limitações e comprometimentos da saúde.

Neste trabalho não foi observada associação estatisticamente significativa entre autoavaliação de saúde e classificação de fragilidade, diferentemente de outros estudos ${ }^{29,37}$. Possivelmente, isso se deu devido à classificação do fenótipo frágil ser dependente do conjunto de outros componentes físicos (baixo nível de atividade física; perda de peso e fraqueza muscular), os quais não mostraram relações com percepção da saúde em nossos resultados. Porém, quando analisados separadamente, alguns componentes se destacaram em termos comparativos.

As limitações deste estudo concernem no número elevado de óbitos ocorridos entre as fases de avaliação e reavaliação, resultando na amostra reduzida para o seguimento. Outro aspecto a ser elucidado foi o processo de amostragem realizado, uma vez que este pode levar um viés de seleção inviabilizando a generalização dos dados para a população.

No entanto, este estudo aborda questões relevantes a respeito da relação entre as medidas subjetivas em saúde e as condições clínicas, os sinais e sintomas clínicos comumente vivenciados e relatados por idosos atendidos em serviços de Geriatria, subsidiando a concepção de que o julgamento da própria saúde condiz com o diagnóstico e/ou exame e transparece o estado de saúde do paciente; além de ser um instrumento de valia em prática clínica e pesquisas, a fim de nortear ações em promoção de saúde e bem-estar das pessoas.

\section{CONCLUSÃO}

A presente pesquisa mostrou a associação entre autoavaliação de saúde e componentes de fragilidade física em idosos ambulatoriais. De um modo geral, os resultados explanados reforçam a necessidade da utilização de medidas subjetivas na Avaliação Geriátrica Ampla, para o rastreio de vulnerabilidades na baixa complexidade em saúde. A relação entre a percepção da própria saúde e a síndrome de fragilidade também avigora a boa concordância entre subjetividade em saúde e condições clínicas ou sinais e sintomas, levan- do em consideração aspectos individuais e valorativos de cada ser humano.

\section{REFERÊNCIAS}

1. Peres MA, Masiero AV, Longo GZ, da Rocha GC, Matos IB, Najnie K, et al. Autoavaliação da saúde em adultos no Sul do Brasil. Rev Saúde Pública. 2010;44(5):901-11. DOI: 10.1590/S0034-89102010000500016

2. Borim F, Barros M, Neri A. Autoavaliação da saúde em idosos: pesquisa debase populacional no Município de Campinas, São Paulo, Brasil. Cad Saúde Pública. 2012;28(4):76980. DOI: 10.1590/S0102-311X2012000400016

3. Borim, FSA, Neri AL, Francisco PMSB, Barros MBD. Dimensões da autoavaliação de saúde em idosos. Rev Saúde Pública. 2014;48(5). DOI: 10.1590/S0034-8910.2014048005243

4. Martinez DJ, Kasl SV, Gill TM, Barry LC. Longitudinal association between self-rated health and timed gait among older persons. J Gerontol B: Psychol Sci Soc Sci. 2009;65(6):715-9. DOI: 10.1093/geronb/gbp115

5. Lima MG, Barros MB, César CL, Goldbaum M, Carandina L, Ciconelli RM. Impact of chronic disease on quality of life among the elderly in the state of São Paulo, Brazil: a population-based study. Rev Panam Salud Pública. 2009;25(4):314-21. DOI: 10.1590/s1020-49892009000400005

6. Moreno X, Albala C, Lera L, Sánchez H, Fuentes-García $\mathrm{A}$, Dangour AD. The role of gender in the association between self-rated health and mortality among older adults in Santiago, Chile: A cohort study. PLoS One. 2017; 12(7):e0181317. DOI: 10.1371/journal.pone.0181317

7. Dapp U, Minder CE, Anders J, Golgert S, von Renteln-Kruse W. Long-term prediction of changes in health status, frailty, nursing care and mortality in community-dwelling senior citizens-results from the longitudinal urban cohort ageing study (LUCAS). BMC Geriatr. 2014;14(1):141. DOI: 10.1186/1471-2318-14-141.

8. Jerez-Roig J, Souza DL, Andrade FL, Lima BF Filho, Medeiros RJ, Oliveira NP, et al. Self-perceived health in institutionalized elderly. Cien Saude Colet. 2016;21(11):336775. DOI: 10.1590/1413812320152111.15562015

9. BezJPO, Neri AL. Velocidade da marcha, força de preensão e saúde percebida em idosos: dados da rede FIBRA Campinas, São Paulo, Brasil. Cien Saúde Colet. 2014;19(8):334353. DOI: 10.1590/1413-81232014198.09592013 
10. Gijón-Conde T, Graciani A, López-García E, García-Esquinas E, Laclaustra M, Ruilope LM, et al. Frailty, disability, and ambulatory blood pressure in older adults. J Am Med Dir Assoc. 2017. DOI: 10.1016/j.jamda.2017.11.014

11. Fried LP, Tangen CM, Walston J, Newman AB, Hirsch C, Gottdiener J, et al. Frailty in older adults: evidence for a phenotype. J Gerontol A Biol Sci Med Sci. 2001;56(3):M146-M57. DOI: 10.1093/gerona/56.3.M146

12. Borim FSA, Barros MBA, Yassuda MS, Eulalio MC. Avaliação subjetiva de saúde e fragilidade. In: Neri AL. Fragilidade e Qualidade de Vida na Velhice. Alínea ed 2013. p. 227-45.

13. Bertolucci PH, Brucki SM, Campacci SR, Juliano Y. The Mini-Mental State Examination in a general population: impact of educational status. Arq Neuropsiquiatr. 1994;52(1):1-7.

14. Radloff LS. The CES-D scale a self-report depression scale for research in the general population. Applied psychological measurement. 1977;1(3):385-401. DOI: $10.1177 / 014662167700100306$

15. Batistoni SST, Neri AL, Cupertino APFB. Validity of the center for epidemiological studies depression scale among Brazilian elderly. Rev Saude Publica. 2007;41(4):598-605. DOI: 10.1590/S0034-89102007000400014

16. Guralnik JM, Simonsick EM, Ferrucci L, Glynn RJ, Berkman LF, Blazer DG, et al. A short physical performance battery assessing lower extremity function: association with self-reported disability and prediction of mortality and nursing home admission. J Gerontol. 1994;49(2): M85-M94. DOI: 10.1093/geronj/49.2.M85

17. Nakano M. Adaptação cultural do instrumento Short Physical Performance Battery-SPPB: adaptação cultural e estudo da confiabilidade [dissertação]. Campinas: Universidade Estadual de Campinas. 2007.

18. Silva V. Desfechos da Síndrome da Fragilidade: um estudo longitudinal com idosos em atendimento ambulatorial [Tese Doutorado]. Universidade Estadual de Campinas: Campinas, SP; 2014.

19. Tavares Batistoni SS, Martins Prestes S, Cachioni M, Vieira da Silva Falcão D, Lopes A, Sanches Yassuda $M$, et al. Categorização e identificação etária em uma amostra de idosos brasileiros residentes na comunidade. Psicologia: Reflexão e Crítica. 2015;28(3). DOI: $10.1590 / 1678-7153.201528310$

20. Passarelli Mantovani E, de Lucca SR, Liberalesso Neri A. Associações entre significados de velhice e bem-estar subjetivo indicado por satisfação em idosos. Rev Bras Geriatr Gerontol. 2016;19(2):203-222. DOI: 10.1590/1809-98232016019.150041

21. Oliveira M, Daher D, Silva J, Andrade S. A saúde do homem em questão: busca por atendimento na atenção básica de saúde. Cien Saude Colet. 2015;20(1):273-8. DOI: 10.1590/1413-81232014201.21732013

22. Manfio Liberalesso TE, Dallazen F, Casali Bandeira VA, Moraes Berlezi E. Prevalência de fragilidade em uma população de longevos na região Sul do Brasil. Saúde em Debate. 2017;41(113). DOI: 10.1590/0103-1104201711316

23. Küchemann BA. Envelhecimento populacional, cuidado e cidadania: velhos dilemas e novos desafios. Sociedade e Estado. 2012;27(1):165-80.

24. doCensoDemográficoIS.RiodeJaneiro:2011.wwwibgegov $\mathrm{br//estatistica//censo2010/} \mathrm{acesso} \mathrm{em.} \mathrm{2010;20(04):2014.}$ DOI: $10.1590 /$ S0102-69922012000100010

25. Sutton JL, Gould RL, Daley S, Coulson MC, Ward EV, Butler AM, et al. Psychometric properties of multicomponent tools designed to assess frailty in older adults: A systematic review. BMC Geriatr. 2016;16(1):55. DOI: $10.1186 / \mathrm{s} 12877-016-0225-2$

26. Leme DE, Thomaz RP, Borim Fs, Brenelli SL, de Oliveira DV, Fattori A. Estudo do impacto da fragilidade, multimorbidade e incapacidade funcional na sobrevida de idosos ambulatoriais. Cien Saude Colet. 2019;24(1):137-146. DOI: 10.1590/1413-81232018241.04952017.

27. Freitas CV, Sarges EdSNF, Moreira KECS, Carneiro SR. Avaliação de fragilidade, capacidade funcional e qualidade de vida dos idosos atendidos no ambulatório de geriatria de um hospital universitário. Rev Bras Geriatr Gerontol. 2016;19(1):119-28. DOI: 10.1590/1809-9823.2016.14244

28. de Carvalho Mello A, Engstrom EM, Alves LC. Fatores sociodemográficos e de saúde associados à fragilidade em idosos: uma revisão sistemática de literatura. Cad Saúde Pública. 2014;30(6):1-25. DOI: 10.1590/0102-311X00148213

29. Melo DM, Falsarella GR, Neri AL. Autoavaliação de saúde, envolvimento social e fragilidade em idosos ambulatoriais. Rev Bras Geriatr Gerontol. 2014;17(3):471-84. DOI: $10.1590 / 1809-9823.2014 .13172$

30. Pérez-Fuentes MC, Molero MM, Mercader I, Soler Flores FJ, Barragán A, Calzadilla Y, et al. Salud percibida y salud real: prevalencia en las personas mayores de 60 años. Enferm Univ.2015;12(2):56-62. DOI: $10.1016 /$ j.reu.2015.03.002

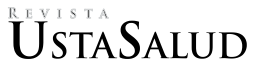


31. Sargent-Cox KA, Anstey KJ, Luszcz MA. Patterns of longitudinal change in older adults' self-rated health: the effect of the point of reference. Health Psychol. 2010;29(2):14352. DOI: $10.1037 / \mathrm{a} 0017652$

32. Reile R, Leinsalu M. Ethnic variation in self-rated healthmortality association: Results from a 17-year follow-up study in Estonia. Medicina (Kaunas). 2017;53(2):114121. DOI: 10.1016/j.medici.2017.04.003

33. Link BG, Susser ES, Factor-Litvak P, March D, Kezios KL, Lovasi GS, et al. Disparities in self-rated health across generations and through the life course. Soc Sci Med. 2017; 174:17-25. DOI: 10.1016/j.socscimed.2016.11.035

34. Siqueira FV, Facchini LA, Piccini RX, Tomasi E, Thumé E, Silveira DSd, et al. Atividade física em adultos e idosos residentes em áreas de abrangência de unidades básicas de saúde de municípios das regiões Sul e Nordeste do Brasil. Cad Saúde Pública. 2008;24(1):39-54. DOI: $10.1590 /$ S0102-311X2008000100005
35. Ebrahimi Z, Wilhelmson K, Eklund K, Moore CD, Jakobsson A. Health despite frailty: exploring influences on frail older adults' experiences of health. Geriatr Nurs. 2013; 34(4):289-94. DOI: 10.1016/j.gerinurse.2013.04.008

36. Eloranta S, Arve S, Isoaho H, Lehtonen A, Viitanen M. Factors connected with positive life orientation at age 70 , 80, 85 and 90-The Turku Elderly Study. Scan J Caring Sci. 2015; 29(3):537-47. DOI: 10.1111/scs.12188

37. Pinto JM, Neri AL. Relações entre níveis de participação social, autoavaliação de saúde e satisfação com a vida em idosos de acordo com gênero. Revista Kairós: Gerontologia. 2016;19(4):255-72. DOI: 10.23925/2176-901X.2016v19i4p255-272

\section{Correos electrónicos de los autores:}

Dayane Capra de Oliveira: dayacapra@gmail.com Giovana Sposito: giovanasposito@gmail.com

Vanessa Abreu da Silva: vanisabreu@hotmail.com Daniel Eduardo da Cunha Leme: daniel.eduardo.7@hotmail.com Maria José D’Elboux: mariadio@uol.com.br 